\section{Comments on a Recently Published PCR Method for Site-Directed Mutagenesis}

I would like to outline the similarities between a polymerase chain reaction (PCR)-based site-directed mutagenesis procedure published by $\mathrm{Xu}$ et al. in BioTechniques (3) and a similar procedure published by me and co-workers a few years ago in Nucleic Acids Research (1).

Both the procedures have common experimental steps, such as performing an inverse PCR on a PCR-generated DNA fragment in which a site-directed mutagenesis is targeted. Inverse PCR was performed using back-to-back (in- ternal) primers, one or both of which may contain desired mutation(s). Inverse PCR products now containing the mutation(s) were then ligated and inserted into a vector, and sequencing was performed to assess the site-directed mutagenesis. Although, some minor differences exist between these two procedures. For example, Xu et al. design their initial PCR primers to their target DNA from a specific area of DNA sequence carrying the desirable restriction sites. Whereas, in our method, primers can be chosen from any area of target DNA molecule, and the desirable restriction sites were tagged to $5^{\prime}$ end of primers, for eventual cloning purposes. $\mathrm{Xu}$ et al. perform inverse PCR on monomeric DNA molecule, and our procedure used concatamers. Using concatamers over monomeric DNA has certain advantages, such as concatamers provide more template for PCRs, and length of original DNA fragment in which a mutations is desired can be maintained. Xu et al. have phosphorylated their back-to-back primers, whereas we have phosphorylated the whole PCR-DNA generated by first inverse PCR for the common purpose, i.e., to prevent mis-orientation during the subsequent ligation step. In addition to phosphorylating the PCR-generated DNA, we have subjected it to the endflushing with Klenow DNA polymerase to prevent addition of non-template base pairs that may occur during PCRs (2).

In brief, considering the minor differences described above, the procedures by $\mathrm{Xu}$ et al. neither offer any new addition nor any significant improvement to our previously described procedure of site-directed mutagenesis by PCR (1). Further, I regret the inability of Xu et al. to quote our reference (1).

\section{REFERENCES}

1.Heda, G.D., T.R. Henion and U. Galili. 1992. A simple in vitro site directed mutagenesis of concatamerized cDNA by inverse polymerase chain reaction. Nucleic Acids Res. 20:5241-5242.

2.Hemsley, A., N. Arnheim, M.D. Toney, G. Cortopassi and D.J. Galas. 1989. A simple method for site-directed mutagenesis using the polymerase chain reaction. Nucleic Acids Res. 17:6545-6551.
3.Xu, X., S.-H. Kang, O. Heidenreich, Q. Li and M. Nerenberg. 1996. Rapid PCR method for site-directed mutagenesis on double-stranded plasmid DNA. BioTechniques 20:44-47.

\author{
Ghanshyam D. Heda \\ Research Service-151 \\ Department of Veterans Affairs \\ Medical Center \\ 1030 Jefferson Avenue \\ Memphis, TN 38104, USA
}

\section{Response to Comments by G.D. Heda}

We regret having overlooked the paper by G.D. Heda, et al. in Nucleic Acids Research (1). As the author points out, this paper does describe directional insertion of mutations by reverse polymerase chain reaction (PCR). However, our paper (2) provides a greatly simplified approach to mutagenesis, does not require concatamerization or purification of concatamerized molecules from unligated precursors and allows mutagenesis directly from genomic DNA.

\section{REFERENCES}

1.Heda, G.D., T.R. Henion and U. Galili. 1992. A simple in vitro site directed mutagenesis of concatamerized cDNA by inverse polymerase chain reaction. Nucleic Acids Res. 20:5241-5242.

2.Xu, X., S.H. Kang, O. Heidenreich, Q. Li and M. Nerenberg. 1996. Rapid PCR method for site-directed mutagenesis on double-stranded plasmid DNA. BioTechniques 20:44-47.

\section{Michael Nerenberg The Scripps Research Institute 10666 North Torrey Pines Road Cal-1 \\ La Jolla, CA 92037, USA}

